\title{
Wideband self-injection-locked green tunable laser diode
}

\author{
M. Hosne M. Shamim' ${ }^{1 \dagger}$ Tien Khee $\mathrm{Ng}^{2}$, Boon S. $\mathrm{Ooi}^{2}$, M. Z. M. Khan ${ }^{1 *}$ \\ ${ }^{I}$ Optoelectronic Research Laboratory, Electrical Engineering Department, King Fahd University of Petroleum \& Minerals (KFUPM), \\ Dhahran 31261, Saudi Arabia \\ ${ }^{2}$ Computer, Electrical and Mathematical Sciences and Engineering (CEMSE) division, King Abdullah University of Science \& Technology \\ (KAUST), Thuwal 23955-6900, Saudi Arabia \\ zahedmk@kfupm.edu.sa
}

\begin{abstract}
A wideband tunability of $6.53 \mathrm{~nm}$ with appreciable SMSR $(>10 \mathrm{~dB})$ and linewidth $(\sim 0.1 \mathrm{~nm})$ is demonstrated from a simple and low-cost tunable self-injection locked InGaN/GaN green laser based external-cavity system, for the first time.

OCIS codes: (140.3520) Lasers, injection-locked; (140.5960) Semiconductor lasers; (140.3600) Lasers, tunable; (140.7300) Visible lasers
\end{abstract}

\section{Introduction}

The typical emission spectrum and the lack of wavelength-tuning investigations of InGaN/GaN laser diodes in the green emission regime forbid the applications green lasers for applications beyond lighting applications. A narrow linewidth tunable laser in the green wavelengths is essential for applications in wavelength-division multiplexed optical wireless communications, spectroscopy, holographic displays, $\mathrm{Sr}+$ ion trapping for $\mathrm{Sr}+$ clocking, or biophotonics. In order to explore techniques for wavelength-tuning, we noted that several methods of attaining wavelength tunability in the near-infrared regime were reported. The methods include monolithic tunable systems employing distributed feedback (DFB) [1] and distributed Bragg reflector (DBR) [2] lasers, albeit with limited tunability. For the visible wavelengths counterpart, external-cavity diode laser (ECDL) system were employed, such as that utilizing the Littrow configuration for wideband tunability [3], [4]. In such configuration, a diffraction grating is typically used to disperse the laser beam into different orders, wherein the zeroth order beam is used as the output and the first order beam as the feedback. There is only one controlling parameter, which is the angle of the diffraction grating that is prone to mis-alignment, and a trade-off between grating efficiency and tunability is inevitable. An alternative Littman-Metcalf configuration solves the misalignment problem after the addition of an extra mirror at the expense of system complexity. In both cases, the operating injection current are usually kept near the threshold current to ensure single longitudinal mode operation while shifting the wavelengths, and thereby limiting the tuning-capacity. Recently, a self-injection locking scheme in visible wavelengths showed great promise obtaining a narrow linewidth emission as small as $0.07 \mathrm{~nm}$ at the linear regime of the biasing current [5]. In this work, such kind of assisting technique is used in an external cavity configuration achieving the desired wideband wavelength tunability and narrow mode linewidth. A comprehensive analysis of the side-mode-suppression-ratio (SMSR) and linewidth at various injection currents is also discussed.

\section{Experimental setup}

Fig. 1 (a) shows the experimental setup of the green tunable laser diode system in a free space configuration. Off the shelf InGaN/GaN laser diode (Thorlabs L520P50) with a maximum optical power of $50 \mathrm{~mW}$ was mounted on (Thorlabs TCLDM9) laser diode mount. The green lasing emission ( 521-528 nm), was then collimated by an aspheric lens (Thorlabs, A110TM-A) and a 92:8\% pellicle beamsplitter (Thorlabs, BP108) splits the light beam orthogonally. The $92 \%$ transmitted light beam travel further before being reflected from a partial mirror (polka dot beamsplitter, Edmund optics) of 30\% reflectivity that was mounted on a kinematic mount (Thorlabs, KM100) and a linear stage, forming an external cavity of $28 \mathrm{~cm}$ length between the laser facet and the partial mirror. Hence, the external cavity length could be tuned along the optical axis and act as a tuning parameter. The transmitted beam from the partial reflector was the usable optical power. The other $8 \%$ of the light beam which was reflected at a right angle of the partial reflector passed through a bi-convex lens (Thorlabs, LB1471-A-ML) of focal length $50 \mathrm{~mm}$ and went into the optical spectrum analyzer (OSA, Yokogawa AQ6373B) of $0.02 \mathrm{~nm}$ resolution for the measurement purpose. The entire experiment was conducted under a precise $20^{\circ} \mathrm{C}$ room temperature.

\footnotetext{
${ }^{\dagger}$ Currently affiliated with Department of Electrical and Computer Engineering, McGill University, Canada.
} 
(a)

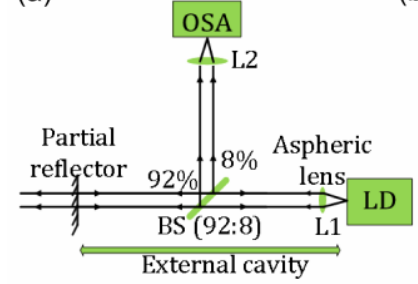

(b)

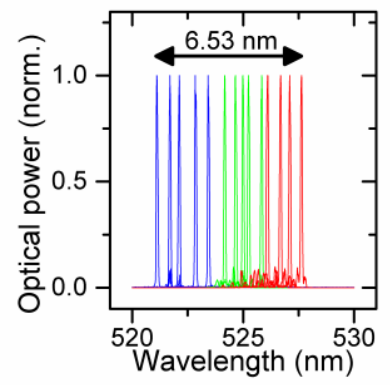

(c)

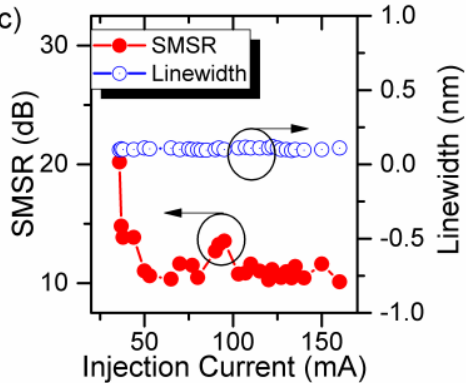

Fig. 1 (a) Block diagram of the self-injection locked tunable laser system, (b) overlapped tunable lasing spectrums showing the tunability of the system at $36 \mathrm{~mA}$ (blue), $95 \mathrm{~mA}$ (green), and $160 \mathrm{~mA}$ (red), and (c) SMSR (red circle) and linewidth (blue circle with a dot) of the locked modes at various injection currents.

\section{Results and discussions}

The laser diode cavity and the external cavity forms a composite cavity that allows longitudinal modes of different free spectral ranges. If the round-trip phase of a certain longitudinal mode matches with that of the composite cavity, locking takes place. The locked mode accumulates all the energy from its neighboring modes raising its peak while suppressing the other modes. Fig. 1 (b) shows such self-locked longitudinal modes at different wavelengths of the green emission spectrum from $521.10 \mathrm{~nm}$ to $527.63 \mathrm{~nm}$. A wideband tunability of $6.53 \mathrm{~nm}$ was achieved from the contribution of $36 \mathrm{~mA}, 95 \mathrm{~mA}$, and $160 \mathrm{~mA}$ injection currents. The single-longitudinal-mode operation is possible at other injection currents as well, and at a much higher resolution than it appears in the spectrum of Fig. 1(b). It is to be noted that, varying temperature would yield more tuning span thus providing a larger tunability as we have observed previously in [6]. The SMSR and the linewidth (full-width-at-half-maximum) of the locked modes at various injection currents have been analyzed and shown in Fig. 1(c). Note that the SMSR and linewidth values corresponds to an average value of all possible locked modes at that particular injection current. The SMSR at the lower injection currents (near to the threshold of $36 \mathrm{~mA}$ ) are higher and reduces significantly at around $1.22 \mathrm{I}_{\mathrm{th}}$ $1.47 \mathrm{I}_{\mathrm{th}}$. The highest SMSR of $30.1 \mathrm{~dB}$ was, however, observed at $75 \mathrm{~mA}\left(2.08 \mathrm{I}_{\mathrm{th}}\right)$ owing to an excellent phase matching condition in the composite cavity. Through the entire tuning range, the minimum SMSR was kept being 10 $\mathrm{dB}$, thus the tuning window is estimated to be larger than the reported value has this threshold is not set. On the other hand, the linewidth, is found to be very consistent across the injection currents at $0.1 \pm 0.02 \mathrm{~nm}$ value, implying a very good stability and affirms the usage of any injection current to bias the laser diode for tuning purpose.

\section{Conclusions}

Self-injection locking technique shows a great promise to develop a visible tunable laser system and thus addressing the issues associated with the conventional tunable systems. A tunability of $6.53 \mathrm{~nm}$ is demonstrated in $\sim 521-528$ $\mathrm{nm}$ with noticeable $>10 \mathrm{~dB}$ SMSR and narrow linewidth of $\sim 0.1 \mathrm{~nm}$. Precise control of the wavelength tuning could be accomplished with a motorized stage and integrated on a single platform making it a compact and user-friendly.

\section{Acknowledgement}

Support from Deanship of Research, KFUPM, through grant KAUST004 is acknowledged. This work is also supported by KAUST grants BAS/1/1614-01-01, REP/1/2878-01-01, KCR/1/2081-01-01, and GEN/1/6607-01-01, and King Abdulaziz City for Science and Technology funding EE2381 and KACST TIC R2-FP-008.

\section{References}

[1] L. Li, S. Tang, J. Lu, Y. Shi, B. Cao, and X. Chen, "Study of cascaded tunable DFB semiconductor laser with wide tuning range and high single mode yield based on equivalent phase shift technique," Opt. Commun., 352, 70-76 (2015).

[2] Y. Dai, K. Xu, J. Wu, Y. Li, X. Hong, H. Guo, and J. Lin, "Design of a monolithic tunable laser based on equivalent-chirp grating reflectors," Opt. Lett., 35, 3880, (2010).

[3] M. Chi, O. B. Jensen, and P. M. Petersen, "Green high-power tunable external-cavity GaN diode laser at 515 nm," Opt. Lett., 41, 4154 (2016).

[4] T. Laurila, T. Joutsenoja, R. Hernberg, and M. Kuittinen, "Tunable external-cavity diode laser at $650 \mathrm{~nm}$ based on a transmission diffraction grating.," Appl. Opt., 41, 5632-5637 (2002).

[5] M. H. M. Shamim, M AShemis, C. Shen, H. M.Oubei, T. K. Ng, B. S. Ooi, and M. Z. M. Khan "Investigation of Self-Injection Locked Visible Laser Diodes for High Bit-Rate Visible Light Communication," IEEE Photonics J., 10, 1-11 (2018).

[6] M. H. M. Shamim, T. K. Ng, B. S. Ooi, and M. Z. M. Khan, “Tunable self-injection locked green laser diode,” Opt. Lett., 43, 4931 (2018). 